\title{
Reverse shock emission driven by post-merger millisecond magnetar winds: Effects of the magnetization parameter
}

\author{
L. D. $\mathrm{Liu}^{1,2}$, L. J. Wang ${ }^{3}$, and Z. G. Dai ${ }^{1,2}$
}

\author{
1 School of Astronomy and Space Science, Nanjing University, 210093 Nanjing, PR China \\ e-mail: dzg@nju.edu.cn \\ 2 Key Laboratory of Modern Astronomy and Astrophysics (Nanjing University), Ministry of Education, 210093 Nanjing, PR China \\ 3 Key Laboratory of Space Astronomy and Technology, National Astronomical Observatories, Chinese Academy of Sciences, \\ 100012 Beijing, PR China
}

Received 4 January 2016 / Accepted 11 April 2016

\begin{abstract}
The study of short-duration gamma-ray bursts provides growing evidence that a good fraction of double neutron star mergers lead to the formation of stable millisecond magnetars. The launch of Poynting flux by the millisecond magnetars could leave distinct electromagnetic signatures that reveal the energy dissipation processes in the magnetar wind. In previous studies, we assume that the magnetar wind becomes completely lepton-dominated so that electrons/positrons in the magnetar wind are accelerated by a diffusive shock. However, theoretical modeling of pulsar wind nebulae shows that in many cases the magnetic field energy in the pulsar wind may be strong enough to suppress diffusive shock acceleration. In this paper, we investigate the reverse shock emission and the forward shock emission with an arbitrary magnetization parameter $\sigma$ of a magnetar wind. We find that the reverse shock emission strongly depends on $\sigma$, and in particular that $\sigma \sim 0.3$ leads to the strongest reverse shock emission. Future observations would be helpful to diagnose the composition of the magnetar wind.
\end{abstract}

Key words. gamma-ray burst: general - radiation mechanisms: non-thermal - pulsars: general

\section{Introduction}

Short-duration gamma-ray bursts (SGRBs) are among the most mysterious phenomena in the universe that have attracted much attention over the past decades. It is generally believed that SGRBs result from compact binary mergers, either double neutron star mergers or neutron star (NS) and black hole (BH) mergers (Paczyński 1986; Eichler et al. 1989; Barthelmy et al. 2005; Fox et al. 2005; Gehrels et al. 2005).

To definitely confirm the compact binary merger nature of SGRBs, next-generation gravitational wave detectors have been coming online recently (Abadie et al. 2010; Bartos et al. 2013). To help localize the gravitational wave sources and determine their redshifts, simultaneous multi-wavelength electromagnetic (EM) detection is crucial. In addition to SGRBs, the neutronrich ejecta unbound during the mergers could power a week-long macronova or kilonova (Li \& Paczyński 1998; Kulkarni 2005; Rosswog 2005; Metzger \& Berger 2012; Berger et al. 2013; Tanvir et al. 2013). The expansion of the ejecta into its ambient medium could lead to radio afterglows (Nakar \& Piran 2011; Metzger \& Berger 2012; Piran et al. 2013; Rosswog et al. 2013).

With the discovery of the X-ray plateau (Rowlinson et al. 2010, 2013) and extended emission, the double neutron star merger is of particular interest because it provides the possibility to inject additional energy (Dai \& Lu 1998a,b; Zhang \& Mészáros 2001) to the ejecta under the assumption that the merger remnant is a magnetar that avoids collapse for an astrophysically interesting period (Dai et al. 2006; Zhang 2013).
Based on the assumption that a stable magnetar is formed following the double neutron star merger, other distinct EM signals could also be observed, e.g., X-ray transients (Zhang 2013), merger-novae (Yu et al. 2013; Metzger \& Piro 2014), and forward-shock afterglows (Gao et al. 2013).

The study of pulsar wind nebulae (PWNe) reveals that an initially Poynting-flux dominated pulsar wind is usually converted to a wind dominated by electron/positron pairs $\left(e^{ \pm}\right)$at some large radii. Under the assumption that the Poynting flux becomes completely lepton dominated (Coroniti 1990; Michel 1994; Dai 2004; Yu \& Dai 2007) and therefore a reverse shock develops within the pulsar wind, an additional EM signature could be observed (Wang \& Dai 2013; Wang et al. 2015). In this case, $e^{ \pm}$pairs in the pulsar wind are accelerated by a diffusive shock.

In fact, the conversion efficiency of the Poynting flux to kinetic flux cannot be $100 \%$. In consequence, the wind may remain strongly magnetized after the acceleration phase. Kennel \& Coroniti (1984) demonstrated that the Crab Nebula's dynamics and emission can be understood provided that the wind is weakly magnetized. There have been a number of analytic and numerical papers on magnetization. The existence of a reverse shock (RS) in magnetized ejecta was suggested (e.g., Fan et al. 2004; Zhang \& Kobayashi 2005; Giannios et al. 2008; Mimica et al. 2009; Lyutikov 2011) and the dynamics of arbitrarily magnetized ejecta-medium interaction were studied (e.g., Zhang \& Kobayashi 2005; Mimica et al. 2009; Mizuno et al. 2009; Lyutikov 2011). It was found that the 
magnetization influences the RS emission (e.g., Mimica et al. 2010; Harrison \& Kobayashi 2013).

In the case of magnetization, the magnetic field in the shocked wind is determined by the shock jump conditions (Kennel \& Coroniti 1984; Fan et al. 2004; Zhang \& Kobayashi 2005; Mao et al. 2010). The RS, arising from the interaction of the wind with the merger ejecta and external medium, depends on the magnetization of the magnetar wind (Fan et al. 2004; Zhang \& Kobayashi 2005; Mimica et al. 2009, 2010; Mao et al. 2010). The strong magnetic fields would suppress the RS in the magnetized wind (Fan et al. 2004; Zhang \& Kobayashi 2005; Giannios et al. 2008; Mimica et al. 2009, 2010).

The aim of this paper is to evaluate effects of the magnetization parameter $\sigma$ on the resulting EM emission. This paper is organized as follows. In Sect. 2, we describe the model in detail and in Sect. 3 we show the results of different dynamical cases. Discussion and conclusions are given in Sect. 4.

\section{Model}

We assume that the merger product is a massive rapidly spinning neutron star. The newborn neutron star may be close to its breakup limit, so we take $P_{0}=1 \mathrm{~ms}$ as the initial spin period. Owing to rapidly differential rotation of the newborn neutron star, the onset of magneto-rotational instability could amplify the magnetic field of such a neutron star to magnetar levels (Duncan \& Thompson 1992; Giacomazzo \& Perna 2013). Hence, a proto-magnetar may have a magnetic field of $\sim 10^{14}-10^{15} \mathrm{G}$. Its spin-down luminosity can be expressed by

$L_{\mathrm{sd}}=L_{\mathrm{sd}, 0}\left(1+\frac{t}{T_{\mathrm{sd}}}\right)^{-2}$,

where the magnetar initial spin-down luminosity $L_{\mathrm{sd}, 0}=1 \times$ $10^{49} \mathrm{erg} \mathrm{s}^{-1} B_{\mathrm{p}, 15}^{2} R_{\mathrm{s}, 6}^{6} P_{0,-3}^{-4}, B_{\mathrm{p}, 15}=B_{\mathrm{p}} / 10^{15} \mathrm{G}$ is the polar-cap dipole magnetic field and $R_{\mathrm{s}, 6}=R_{\mathrm{s}} / 10^{6} \mathrm{~cm}$ is the stellar radius. Here we take the conventional usage $Q=10^{n} Q_{n}$. The characteristic spin-down timescale of the millisecond magnetar is given by

$T_{\mathrm{sd}}=2 \times 10^{3} \mathrm{~s} I \mathrm{~s}, 45 B_{p, 15}^{-2} R_{\mathrm{s}, 6}^{-6} P_{0,-3}^{2}$,

where $I_{\mathrm{s}, 45}=I_{\mathrm{s}} / 10^{45} \mathrm{~g} \mathrm{~cm}^{2}$ is the magnetar moment of inertia. For a millisecond massive magnetar, the typical values of $I_{\mathrm{s}, 45}, R_{\mathrm{s}, 6}$, and $P_{0,-3}$ are all close to unity.

Recently, numerical simulations have shown that the ejecta from neutron star binary mergers have a typical velocity $v_{\mathrm{ej}}=0.1-0.3 \mathrm{c}$ and a typical mass $M_{\mathrm{ej}}=10^{-4}-10^{-2} M_{\odot}$ (Rezzolla et al. 2010; Hotokezaka et al. 2013; Rosswog et al. 2013). The release of millisecond magnetar rotational energy could launch a Poynting-flux dominated outflow whose luminosity is determined by Eq. (1). This outflow would quickly catch up and interact with the ejecta at a radius $R_{\text {ca }} \sim v_{\text {ej }} t_{\text {delay }}=$ $6 \times 10^{10} \mathrm{~cm}\left(v_{\mathrm{ej}} / 0.2 c\right) t_{\text {delay }, 1}$, where $t_{\text {delay }}=10 t_{\text {delay, } 1} \mathrm{~s}$ is the delay time between the merger and the launch of Poyntingflux dominated outflow (Metzger et al. 2011). A forward shock would be driven into the ejecta because the magnetar wind could push the ejecta to a relativistic speed. The forward shock would cross the ejecta on a short timescale (Gao et al. 2013) and then the forward shock propagates into the ambient medium.

Since the fluctuating component of the magnetic field in the outflow can be dissipated by magnetic reconnection, the
Poynting flux is converted to the kinetic energy of the outflow, and the magnetar wind eventually becomes leptonic-matterdominated (Dai 2004; Coroniti 1990; Michel 1994). In this scenario, a reverse shock develops and produces additional radiation signals (Dai 2004; Yu \& Dai 2007; Mao et al. 2010; Wang \& Dai 2013; Wang et al. 2015). In some cases, however, the magnetar wind may remain rather strongly magnetized at large radii. The magnetar wind in these cases is more likely to be a mixture of Poynting-flux and ultra-relativistic $e^{ \pm}$pairs. To quantify the magnetic field component in the outflow, the magnetization parameter $\sigma$, i.e., the ratio of the Poynting flux $F_{\mathrm{P}}$ to the matter flux $F_{\mathrm{m}}$, is introduced

$\sigma \equiv \frac{F_{\mathrm{P}}}{F_{\mathrm{m}}}=\frac{B^{2}}{4 \pi \Gamma \rho c^{2}}=\frac{B^{\prime 2}}{4 \pi \rho^{\prime} c^{2}}$,

where $B$ and $\rho$ are the magnetic field strength and matter density in the observer frame, and $B^{\prime}$ and $\rho^{\prime}$ are the corresponding quantities in the comoving frame of the outflow.

If the magnetar wind is leptonic-matter-dominated, its bulk Lorentz factor $\Gamma_{\mathrm{w}}$ is typically taken to range from $10^{4}$ to $10^{7}$ (Atoyan 1999; Michel \& Li 1999). In principle, the value of $\Gamma_{\mathrm{w}}$ depends on the magnetization parameter $\sigma$. For simplicity, we here adopt $\Gamma_{\mathrm{w}} \sim 10^{4}$ as a fiducial value.

The basic physical picture of our model is schematized in Fig. 1 of Gao et al. (2013) and Fig. 1 of Wang et al. (2015). The interaction of the magnetar wind with the ejecta and ambient medium would give rise to a relativistic wind bubble (Dai 2004; Yu \& Dai 2007; Mao et al. 2010; Wang \& Dai 2013; Wang et al. 2015). Two shocks are formed: a reverse shock that propagates into the cold magnetar wind and a forward shock that propagates into the ambient medium. Thus, the relativistic wind bubble includes four different regions separated by a contact discontinuity surface and two shocks. The four regions are (1) the unshocked ambient medium; (2) the forward-shocked ambient medium; (3) the reverse-shocked magnetar wind; and (4) the unshocked cold wind. Regions 2 and 3 are divided by a contact discontinuity surface. The shocked regions produce synchrotron emission. Hereafter, we denote the quantities of Region $i$ as follows: $n_{i}^{\prime}, B_{i}^{\prime}, e_{i}^{\prime}$, and $P_{i}^{\prime}$ are particle number density, magnetic field strength, energy density, and pressure, where the primes refer to the quantities in the comoving frame and the subscript $i$ represents Regions 1-4.

In general, the ambient medium is unmagnetized so that $\sigma_{1}=0$. The magnetization degree of the magnetar wind could be quantified by the magnetization parameter

$\sigma \equiv \sigma_{4}=\frac{B_{4}^{\prime 2}}{4 \pi n_{4}^{\prime} m_{e} c^{2}}$.

Assuming that the magnetar wind is isotropic, the comoving electron density of the unshocked cold wind is given by

$n_{4}^{\prime}=\frac{L_{0}}{4 \pi R^{2} \Gamma_{4}^{2} m_{e} c^{3}(1+\sigma)}$,

where $L_{0}=\xi L_{\mathrm{sd}, 0}$ with $\xi$ denoting the fraction of the spin-down power injected into the shocks, $R$ the radius of the wind bubble, $\Gamma_{4}$ the Lorentz factor of Region 4 , and in our calculation we take $\Gamma_{4}=\Gamma_{\mathrm{w}}$. Under ideal MHD conditions, owing to the induction equation the magnetic field in the wind is dominated by its toroidal component (Fan et al. 2004; Zhang \& Kobayashi 2005; Mao et al. 2010). Both $n_{4}^{\prime}$ and $B_{4}^{\prime 2}$ would decrease as $R^{-2}$, and as a result the magnetization parameter $\sigma$ can be regarded as a constant. 
The connection of the two sides of the shocks can be described by the shock jump conditions (Coroniti 1990; Zhang \& Kobayashi 2005; Mao et al. 2010)

$$
\begin{aligned}
& \frac{e_{2}^{\prime}}{n_{2}^{\prime} m_{p} c^{2}}=\Gamma_{2}-1, \\
& \frac{n_{2}^{\prime}}{n_{1}}=4 \Gamma_{2}+3, \\
& \frac{e_{3}^{\prime}}{n_{3}^{\prime} m_{e} c^{2}}=\left(\Gamma_{34}-1\right) f_{a}, \\
& \frac{n_{3}^{\prime}}{n_{4}^{\prime}}=\left(4 \Gamma_{34}+3\right) f_{b}, \\
& \frac{B_{3}^{\prime}}{B_{4}^{\prime}}=\left(4 \Gamma_{34}+3\right) f_{b}, \text { for } \sigma>0,
\end{aligned}
$$

where $m_{p}$ is the proton rest mass, $\Gamma_{34} \approx \frac{1}{2}\left(\Gamma_{3} / \Gamma_{4}+\Gamma_{4} / \Gamma_{3}\right)$ is the bulk Lorentz factor of Region 3 measured in the comoving frame of Region 4 , and $f_{a}$ and $f_{b}$ are the correction factors for $e_{3}^{\prime} / n_{3}^{\prime} m_{e} c^{2}$ and $n_{3}^{\prime} / n_{4}^{\prime}$ with respect to the $\sigma=0$ case. When $\Gamma_{34} \gg 1, f_{a}$ and $f_{b}$ can be expressed as (Zhang \& Kobayashi 2005; Mao et al. 2010)

$$
\begin{aligned}
& f_{a} \approx 1-\frac{\sigma}{2\left[u_{3 \mathrm{~s}}^{2}+u_{3 \mathrm{~s}}\left(u_{3 \mathrm{~s}}^{2}+1\right)^{1 / 2}\right]}, \\
& f_{b} \approx \frac{1}{4}\left[1+\left(1+\frac{1}{u_{3 \mathrm{~s}}^{2}}\right)^{1 / 2}\right],
\end{aligned}
$$

where $f_{a} \rightarrow 1$ and $f_{b} \rightarrow 1$ when $\sigma \rightarrow 0$, and $u_{3 s}=\Gamma_{3} \beta_{3}$ is the four-velocity of Region 3 measured in the rest frame comoving with the reverse shock. When $\Gamma_{34} \gg 1, u_{3 \mathrm{~s}}$ can be easily solved analytically from (Fan et al. 2004; Zhang \& Kobayashi 2005)

$8(\sigma+1) u_{3 \mathrm{~s}}^{4}-\left(8 \sigma^{2}+10 \sigma+1\right) u_{3 \mathrm{~s}}^{2}+\sigma^{2}=0$.

Equal speed and pressure equilibrium across the contact discontinuity surface give

$\Gamma_{2}=\Gamma_{3}$,

$P_{3}^{\prime}=P_{\mathrm{th}, 3}^{\prime}+P_{\mathrm{B}, 3}^{\prime}=P_{2}^{\prime}$,

where $P_{\mathrm{th}, 3}^{\prime}$ is the thermal pressure and $P_{\mathrm{B}, 3}^{\prime}$ is the magnetic pressure of Region 3. According to the jump conditions we can obtain $P_{\text {th, } 3}^{\prime}=\frac{1}{3} e_{3}^{\prime} \approx \frac{4}{3} \Gamma_{34}^{2} n_{4}^{\prime} m_{e} c^{2} f_{a} f_{b}$ and $P_{\mathrm{B}, 3}^{\prime}=\frac{B_{3}^{\prime 2}}{8 \pi} \approx$ $8 \Gamma_{34}^{2} n_{4}^{\prime} m_{e} c^{2} \sigma f_{b}^{2}$. The total kinetic energy of Region 2 is approximated by

$E_{\mathrm{k}, 2}=\left(\Gamma_{2}-1\right)\left(M_{\mathrm{ej}}+M_{\mathrm{sw}}\right) c^{2}+\Gamma_{2}\left(\Gamma_{2}-1\right) M_{\mathrm{sw}} c^{2}$,

where $M_{\mathrm{sw}}$ is the swept-up medium mass. Owing to energy conservation, any increase in $E_{\mathrm{k}, 2}$ should be equal to the work done by Region 3,

$\mathrm{d} E_{\mathrm{k}, 2}=\delta W=4 \pi R^{2} P_{3}^{\prime} \mathrm{d} R$.

Then we can obtain

$\frac{\mathrm{d} \Gamma_{2}}{\mathrm{~d} R}=\frac{4 \pi R^{2}}{M_{\mathrm{ej}}+2 \Gamma_{2} M_{\mathrm{sw}}}\left[\frac{P_{3}^{\prime}}{c^{2}}-\left(\Gamma_{2}^{2}-1\right) n_{1} m_{p}\right]$.

The evolutions of the swept-up mass $M_{\mathrm{sw}}$ and the radius $R$ of the shock can be described as (Huang et al. 1999)

$$
\begin{aligned}
& \frac{\mathrm{d} M_{\mathrm{sw}}}{\mathrm{d} R}=4 \pi R^{2} n_{1} m_{p}, \\
& \frac{\mathrm{d} R}{\mathrm{~d} t}=\frac{\beta c}{1-\beta} .
\end{aligned}
$$

The energy injected by the magnetar is deposited in the forward shock and in the reverse shock. In the non-magnetized case, the energy contained in the forward shock is

$E_{\mathrm{fs}} \approx\left(\Gamma^{2}-1\right) M_{\mathrm{sw}} c^{2}$,

which is determined by the shock jump conditions. The energy deposited in reverse shock $E_{\mathrm{rs}}$ is comparable to $E_{\mathrm{fs}}$ (Blandford \& McKee 1976; Wang \& Dai 2013; Wang et al. 2015). In an arbitrarily magnetized wind, the total energy of the wind is in kinetic (electron-positron pairs) and magnetic forms. Their fractions are $\frac{1}{1+\sigma}$ and $\frac{\sigma}{1+\sigma}$, respectively. The pairs in the magnetar wind will be reverse-shocked upon interaction with the ambient medium to gain an energy $\frac{1}{\sigma+1}\left(\Gamma^{2}-1\right) M_{\mathrm{sw}} c^{2}$. Consequently, the total energy contained in the blast wave is $\left(\frac{1}{\sigma+1}+1\right)\left(\Gamma^{2}-1\right) M_{\mathrm{sw}} c^{2}$. The dynamics of the blast wave could be determined by

$L_{0} \min \left(t, T_{\mathrm{sd}}\right)=\left(\Gamma-\Gamma_{\mathrm{ej}, 0}\right) M_{\mathrm{ej}} c^{2}+\left(\frac{\sigma+2}{\sigma+1}\right)\left(\Gamma^{2}-1\right) M_{\mathrm{sw}} c^{2}$,

where $\Gamma$ is the Lorentz factor of the ejecta with initial Lorentz factor $\Gamma_{\text {ej, } 0}$. The dynamic equation in Wang \& Dai (2013) does not take the magnetization into account. Wang \& Dai (2013) adopted the factor of 2 in the second term on the right-hand side. The factor $\frac{\sigma+2}{\sigma+1}$ is reduced to the non-magnetized case when the magnetization parameter $\sigma$ approaches zero. For the Poyntingflux dominated magnetar wind, $\sigma$ is high enough so that there is no reverse shock into the wind. The factor is reduced to the Gao et al. (2013) situation. Therefore, this factor can be used in more general cases.

Initially, $\left(\Gamma-\Gamma_{\mathrm{ej}, 0}\right) M_{\mathrm{ej}} c^{2} \gg\left(\frac{\sigma+2}{\sigma+1}\right)\left(\Gamma^{2}-1\right) M_{\mathrm{sw}} c^{2}$, so that the ejecta is accelerated linearly with time until $t=$ $\min \left(T_{\mathrm{sd}}, T_{\mathrm{dec}}\right)$. The deceleration timescale $T_{\mathrm{dec}}$ is defined by the condition $\left(\Gamma-\Gamma_{\mathrm{ej}, 0}\right) M_{\mathrm{ej}} c^{2}=\left(\frac{\sigma+2}{\sigma+1}\right)\left(\Gamma^{2}-1\right) M_{\mathrm{sw}} c^{2}$. The dynamical evolution of the ejecta depends strongly on the mass of the ejecta $M_{\text {ej }}$. By setting $T_{\text {sd }} \sim T_{\text {dec }}$, we can obtain a critical ejecta mass (Gao et al. 2013; Wang \& Dai 2013; Wang et al. 2015)

$M_{\mathrm{ej}, \mathrm{c}} \sim 10^{-3} M_{\odot} n^{1 / 8} I_{45}^{5 / 4} L_{0,47}^{-3 / 8} P_{0,-3}^{-5 / 2} \xi^{5 / 4}\left(\frac{\sigma+2}{\sigma+1}\right)^{1 / 8}$.

It is this critical mass that divides the dynamics of the ejecta into three cases (Gao et al. 2013; Wang \& Dai 2013; Wang et al. 2015): Case I for $M_{\mathrm{ej}}<M_{\mathrm{ej}, \mathrm{c}}$, which is equivalent to $T_{\mathrm{dec}}<T_{\mathrm{sd}}$, Case II for $T_{\mathrm{dec}}=T_{\mathrm{sd}}$, and Case III for $T_{\mathrm{dec}}>T_{\mathrm{sd}}$. With the propagation of the shocks, the bulk kinetic energy of the ejecta and the rotational energy of the magnetar wind would be gradually transformed into internal energy of the shocked matter. Both the forward shock and the reverse shock could heat the cold materials, and particles could be accelerated by these relativistic shocks. We consider synchrotron radiation of the accelerated electrons in the shocked regions. It is similar to the standard model of gamma-ray burst afterglows, whose spectrum includes four power-law segments separated by three break frequencies, i.e., the self-absorption frequency $v_{\mathrm{a}}$, the characteristic frequency $v_{\mathrm{m}}$, and the cooling frequency $v_{\mathrm{c}}$, with peak flux $F_{v \text {,max }}$ (Sari et al. 1998). We obtain the self-absorption frequency $v_{\mathrm{a}}$ according to Wu et al. (2003).

The forward shock emission is calculated quantitatively in a way similar to Gao et al. (2013). For the reverse-shocked wind, the energy density and the magnetic field can be determined by the shock jump conditions for an arbitrary magnetization parameter $\sigma$. The minimum Lorentz factor of $e^{ \pm}$pairs in the shocked 
wind is

$\gamma_{\mathrm{m}}=\frac{p-2}{p-1} \frac{e_{3}^{\prime}}{n_{3}^{\prime} m_{e} c^{2}}$,

where $p$ is the power-law index of the electron energy distribution in Region 3. To find the maximum Lorentz factor of electrons, two timescales are considered here. The first is the particle acceleration timescale

$t_{\mathrm{acc}}=\frac{\gamma_{e} m_{e} c}{q_{e} B^{\prime}}$.

The second is the electron cooling time

$t_{\mathrm{cool}}=\frac{\gamma_{e} m_{e} c^{2}}{P_{\mathrm{rad}}^{\prime}}$,

where the radiation power is given by

$P_{\mathrm{rad}}^{\prime}=\frac{4}{3} \sigma_{\mathrm{T}} c \gamma_{e}^{2} \frac{B^{\prime 2}}{8 \pi}$.

After identifying these two timescales, we have the maximum Lorentz factor

$\gamma_{\max } \approx\left(\frac{6 \pi q_{e}}{\sigma_{\mathrm{T}} B^{\prime}}\right)^{1 / 2}$.

The cooling Lorentz factor of the electrons accelerated by the reverse shock is given by

$\gamma_{\mathrm{c}}=\frac{6 \pi m_{e} c}{\sigma_{\mathrm{T}} \Gamma_{3} B_{3}^{\prime 2} t}$,

where $\sigma_{\mathrm{T}}$ is the Thompson cross section. Owing to the braking caused by the ejecta, at the beginning the magnetar wind can only drive the ejecta in a small radius, leading to a high energy density and a strong magnetic field in Region 3. Consequently, the cooling of $e^{ \pm}$pairs is so quick that the cooling Lorentz factor $\gamma_{\mathrm{c}} \approx 1$, and this Lorentz factor does not deviate significantly from unity until after $T_{\mathrm{ct}}$ (Wang \& Dai 2013). The fast cooling feature of the reverse shock before $T_{\mathrm{ct}}$ seems to be a concern for the validity of Eq. (20) because this equation is only correct if the upper energy cutoff $\gamma_{\max }$ is much larger than $\gamma_{\mathrm{m}}$. It is found that in our calculation $\gamma_{\max } \gg \gamma_{\mathrm{m}}$ is always satisfied. Thus our results are almost independent of $\gamma_{\max }$. This is true because here we have three timescales: the dynamical timescale $t$, the cooling timescale $t_{\text {cool }}$, and the acceleration timescale $t_{\text {acc }}$. When $t<T_{\mathrm{ct}}$, we have $t_{\text {acc }} \ll t_{\text {cool }} \ll t$, i.e., the electrons can be accelerated to high energy before they are cooled by synchrotron radiation.

At the spin-down time $T_{\text {sd }}$, the central engine turns off and the unshocked wind (Region 4) disappears and thereafter Region 3 begins to spread linearly. Assume that the width of the reverse-shocked wind in the comoving frame $\Delta_{\mathrm{RS}} \propto R$. Before the spin-down time of the magnetar, the total number of sweptup $e^{ \pm}$pairs in the shocked wind is $N_{3, \mathrm{e}}=\frac{L_{0} t}{\Gamma_{4} m_{e} c^{2}(1+\sigma)}$. After $T_{\mathrm{sd}}$, Region 4 does not exist and there are no new accelerated electrons. Hence, the total number of particles in the emission region is a constant. Owing to the spread in the width of Region 3, the number density and energy density decrease. When $t>T_{\text {sd }}$,

$n_{3}^{\prime}=n_{3, \mathrm{sd}}^{\prime} \frac{\Gamma_{\mathrm{sd}}}{\Gamma_{3}}\left(\frac{R_{\mathrm{sd}}}{R}\right)^{3}$

where $n_{3, \mathrm{sd}}^{\prime}, \Gamma_{\mathrm{sd}}$, and $R_{\mathrm{sd}}$ are the number density, Lorentz factor and radius at the spin-down time $T_{\text {sd }}$, respectively. Furthermore, since the $e^{ \pm}$pairs are in the slow cooling regime after $T_{\mathrm{sd}}, \gamma_{\mathrm{m}}$ should keep constant.
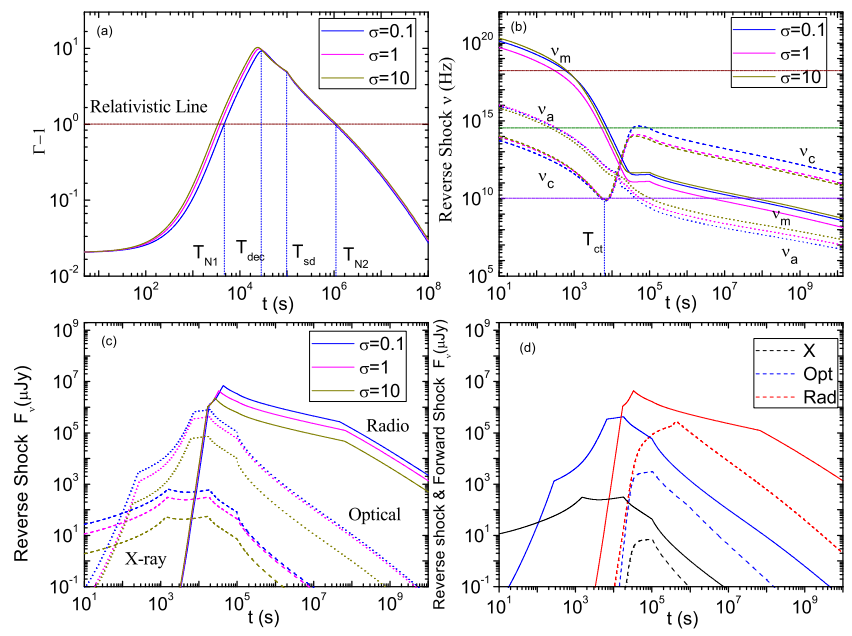

Fig. 1. Calculation results for Case I: $L_{0}=10^{47} \mathrm{erg} \mathrm{s}^{-1}, M_{\mathrm{ej}}=10^{-4} M_{\odot}$, $\xi=0.5, p=2.3$. a) The evolution of the Lorentz factor of the blast wave with different values of $\sigma$ as labeled. b) The break frequencies $v_{\mathrm{a}}$ (dotted), $v_{\mathrm{m}}$ (solid), and $v_{\mathrm{c}}$ (dashed) of the reverse shock emission. The three dashed to dotted lines mark the X-ray, optical $(R)$, and radio $(10 \mathrm{GHz})$ bands, respectively. c) Light curves of the reverse shock in $\mathrm{X}$-ray, optical $(R)$, and radio $(10 \mathrm{GHz})$ bands. d) The solid lines represent the reverse shock light curves for $\sigma=0.1$ and the dashed lines are light curves for the forward shock. In panels a)-c) the blue, magenta, and dark yellow lines represent $\sigma=0.1,1$, and 10, respectively. In panel d), we can obtain that in Case I the reverse shock emission is stronger than the forward shock emission. It can be seen that the X-ray flux lasts for several tens of seconds, while the optical flux maintains a high level until the spin-down time $T_{\text {sd }}$, and then the radio emission becomes dominant.
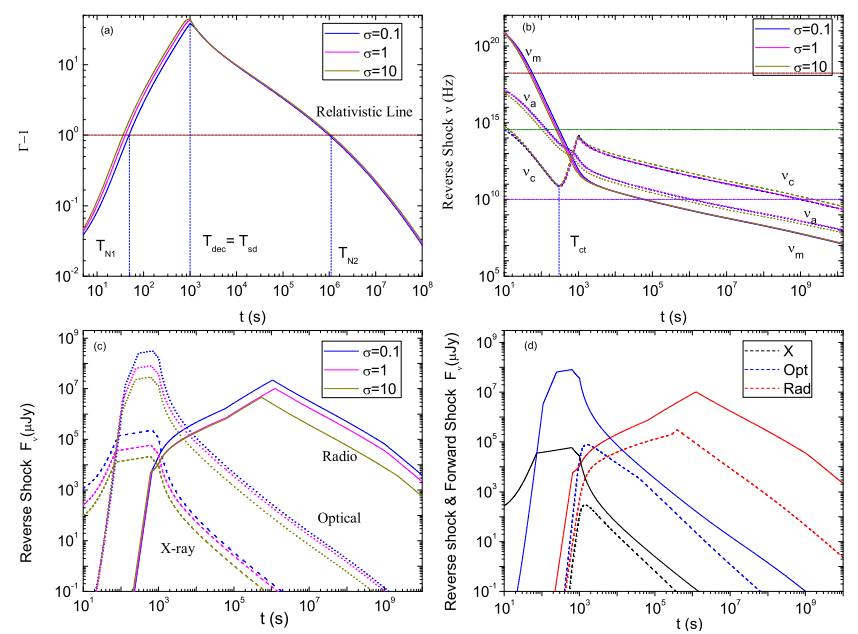

Fig. 2. Calculation results for Case II: $L_{0}=10^{49} \mathrm{erg} \mathrm{s}^{-1}, M_{\mathrm{ej}}=10^{-4} M_{\odot}$. Descriptions of panels are the same as in Fig. 1. In panel a) the blast wave decelerates immediately after linearly accelerating. In panels c) and d), owing to more energy injection to the blast wave than Case I, both reverse shock and forward shock emission is stronger than in Case I.

\section{Results}

For a quantitative analysis, we show the analytical results of the reverse shock based on the dynamic Eq. (18) for the three cases mentioned above. Numerical results including the dynamics, evolution of typical frequencies of the reverse shock, and light curves of emission from the shocked regions obtained by the fourth-order Runge-Kutta method to the dynamic evolution Eqs. (14)-(16) are presented in Figs. 1 to 3. 

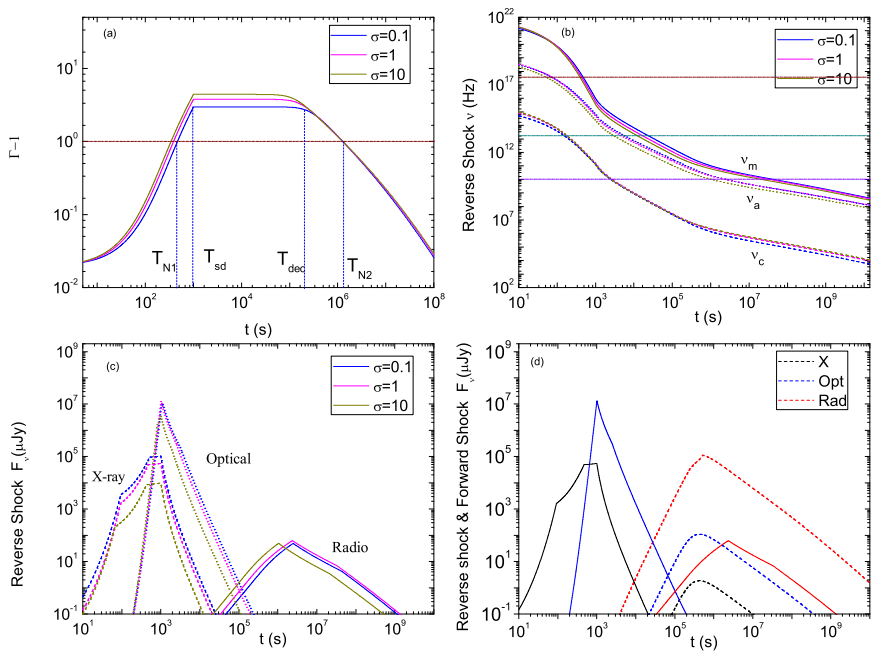

Fig. 3. Calculation results for Case III: $L_{0}=10^{49} \mathrm{erg} \mathrm{s}^{-1}, M_{\mathrm{ej}}=$ $10^{-3} M_{\odot}$. Descriptions of panels are the same as in Fig. 1. In panel a) the dynamic of the blast wave shows a coasting phase between $T_{\text {sd }}$ and $T_{\text {dec }}$. In panel $\mathbf{b}$ ), the break frequencies $v_{\mathrm{a}}, v_{\mathrm{m}}$, and $v_{\mathrm{c}}$ do not intersect each other.

Case I: $M_{\mathrm{ej}}<M_{\mathrm{ej}, \mathrm{c}}$, or $T_{\mathrm{dec}}<T_{\mathrm{sd}}$. According to Eq. (19) $M_{\mathrm{ej}, \mathrm{c}} \propto L_{0}^{-3 / 8}$, so we consider a small $L_{0}$ and a small $M_{\mathrm{ej}}$ to satisfy $M_{\mathrm{ej}}<M_{\mathrm{ej}, \mathrm{c}}$. We take the same parameters as Gao et al. (2013) $L_{0}=10^{47} \mathrm{erg} \mathrm{s}^{-1}$ and $M_{\mathrm{ej}}=10^{-4} M_{\odot}$. In order to describe the dynamics in this case, we need to list the characteristic timescales of the blast wave dynamics and the Lorentz factor at the deceleration time (Gao et al. 2013; Wang \& Dai 2013; Wang et al. 2015)

$T_{\mathrm{N} 1}=2.07 \times 10^{-2}$ days $L_{0,47}^{-1} M_{\mathrm{ej},-4}$,

$T_{\mathrm{dec}}=0.29$ days $L_{0,47}^{-7 / 10} M_{\mathrm{ej},-4}^{4 / 5} n^{-1 / 10}\left(\frac{\sigma+2}{\sigma+1}\right)^{7 / 10}$,

$T_{\text {sd }}=2.3$ days $L_{0,47}^{-1}$,

$T_{\mathrm{N} 2}=28.58$ days $L_{0,47}^{1 / 3} T_{\text {sd }, 5}^{1 / 3} n^{-1 / 3}$,

$\Gamma_{\mathrm{dec}}=7.17 L_{0,47}^{3 / 10} M_{\mathrm{ej},-4}^{-1 / 5} n^{-1 / 10}\left(\frac{\sigma+2}{\sigma+1}\right)^{-3 / 10}+1$,

where $T_{\mathrm{N} 1}$ is the timescale of the blast wave evolving from the non-relativistic to the relativistic phase due to energy injection, which is defined by $\gamma-1=1$, and $T_{\mathrm{N} 2}$ is the timescale of the blast wave evolving from the relativistic to non-relativistic phase due to deceleration by the ambient medium.

Although the dynamical evolution is almost insensitive to the magnetization parameter $\sigma$, the magnetic field in Region 3 is predominantly due to the compression of the upstream magnetic field so that the $\sigma$ of the unshocked wind determines the magnetic field strength of the reverse shock. In consequence, the $\sigma$ value could affect the characteristic timescales and the break frequencies of the synchrotron radiation,

$$
\begin{aligned}
& T_{\mathrm{ct}}=3.2 \times 10^{-3} \text { days } L_{0,49}^{-2 / 3} M_{\mathrm{ej},-4}^{5 / 6} f_{b}^{1 / 3}\left(\frac{\sigma}{1+\sigma}\right)^{1 / 6}, \\
& T_{\mathrm{ac}}=4.1 \times 10^{-3} \text { days } c_{1}^{1 / 23} L_{0,49}^{-31 / 46} n_{1}^{1 / 23} M_{\mathrm{ej},-4}^{20 / 23} f_{b}^{9 / 23}\left(\frac{\sigma}{1+\sigma}\right)^{9 / 46},
\end{aligned}
$$$$
T_{\mathrm{mc}}=6.6 \times 10^{-3} \text { days } L_{0,49}^{-5 / 7} M_{\mathrm{ej},-4}^{6 / 7} \varepsilon_{e}^{1 / 7} \Gamma_{4,4}^{1 / 7} f_{b}^{2 / 7}\left(\frac{\sigma}{1+\sigma}\right)^{1 / 7},
$$

where $T_{\mathrm{ac}}$ and $T_{\mathrm{mc}}$ are two critical times when the cooling frequency $v_{\mathrm{c}}$ crosses the self-absorption frequency $v_{\mathrm{a}}$ and the characteristic frequency $v_{\mathrm{m}}$, respectively.

The break frequencies and peak flux of Region 3 at $T_{\mathrm{dec}}$ are derived as

$$
\begin{aligned}
v_{\mathrm{a}, \mathrm{dec}}= & 2.5 \times 10^{9} \mathrm{~Hz} c_{1}^{3 / 5} L_{0,47}^{33 / 50} M_{\mathrm{ej},-4}^{-6 / 25} \Gamma_{4,4}^{-1} n^{19 / 50} f_{a}^{-1} f_{b}^{2 / 5} \\
& \times\left(\frac{p-1}{p-2}\right)\left(\frac{\sigma}{1+\sigma}\right)^{1 / 5}\left(\frac{\sigma+1}{\sigma+2}\right)^{67 / 200}, \\
v_{\mathrm{m}, \mathrm{dec}}= & 3.14 \times 10^{13} \mathrm{~Hz} \Gamma_{4,4}^{2} n^{1 / 2} f_{a}^{2} f_{b}\left(\frac{p-2}{p-1}\right)^{2} \\
\times & \sqrt{\frac{\sigma}{(1+\sigma)^{5}}\left(\frac{\sigma+1}{\sigma+2}\right)^{1 / 8},} \\
v_{\mathrm{c}, \mathrm{dec}}= & 1.1 \times 10^{13} \mathrm{~Hz} L_{0,47}^{1 / 5} M_{\mathrm{ej},-4}^{-4 / 5} n^{-9 / 10} f_{b}^{-3} \\
\times & \left(\frac{1+\sigma)^{3 / 2}\left(\frac{\sigma+1}{\sigma+2}\right)^{23 / 40},}{\sigma},\right. \\
F_{v, \text { max }, \mathrm{dec}}= & 2.2 \times 10^{4} \mathrm{mJy} L_{0,47}^{9 / 10} M_{\mathrm{ej},-4}^{2 / 5} \Gamma_{4,4}^{-1} n^{1 / 5} D_{27}^{-2} f_{b} \\
& \times \frac{\sqrt{\sigma}}{(1+\sigma)^{3 / 2}}\left(\frac{\sigma+2}{\sigma+1}\right)^{9 / 40},
\end{aligned}
$$

where $c_{1}=\frac{16 \times 2^{2 / 3}}{3 \Gamma(1 / 3)} \frac{p+2}{3 p+2}\left(\mathrm{Wu}\right.$ et al. 2003) and $D_{27}$ is the luminosity distance to the source in units of $10^{27} \mathrm{~cm}$.

Case II: $M_{\mathrm{ej}}=M_{\mathrm{ej}, \mathrm{c}}$, or $T_{\mathrm{dec}}=T_{\mathrm{sd}}$. We adopt $L_{0}=$ $10^{49} \mathrm{erg} \mathrm{s}^{-1}$ and $M_{\mathrm{ej}}=10^{-4} M_{\odot}$, which satisfies $T_{\mathrm{dec}}=$ $T_{\text {sd }}$. The characteristic timescales of the blast wave dynamics and the Lorentz factor at the spin-down time (Gao et al. 2013; Wang \& Dai 2013; Wang et al. 2015)

$$
\begin{aligned}
& T_{\mathrm{N} 1}=2.07 \times 10^{-4} \text { days } L_{0,49}^{-1} M_{\mathrm{ej},-4}, \\
& T_{\mathrm{dec}}=T_{\mathrm{sd}}=2.3 \times 10^{-2} \text { days } L_{0,49}^{-1}, \\
& T_{\mathrm{N} 2}=28.58 \text { days } L_{0,49}^{1 / 3} T_{\mathrm{sd}, 3}^{1 / 3} n^{-1 / 3} \\
& \Gamma_{\mathrm{dec}}=55.9 L_{0,49} T_{\mathrm{sd}, 3} M_{\mathrm{ej},-4}^{-1}+1 \\
& T_{\mathrm{ct}}=3.2 \times 10^{-3} \text { days } L_{0,49}^{-2 / 3} M_{\mathrm{ej},-4}^{5 / 6} f_{b}^{1 / 3}\left(\frac{\sigma}{1+\sigma}\right)^{1 / 6}, \\
& T_{\mathrm{ac}}=4.1 \times 10^{-3} \text { days } c_{1}^{1 / 23} L_{0,49}^{-31 / 46} n_{1}^{1 / 23} M_{\mathrm{ej},-4}^{20 / 23} f_{b}^{9 / 23}\left(\frac{\sigma}{1+\sigma}\right)^{9 / 46}, \\
& T_{\mathrm{mc}}=6.6 \times 10^{-3} \text { days } L_{0,49}^{-5 / 7} M_{\mathrm{ej},-4}^{6 / 7} \Gamma_{4,4}^{1 / 7} f_{a}^{1 / 7} f_{b}^{2 / 7}\left(\frac{\sigma}{1+\sigma}\right)^{1 / 7} .
\end{aligned}
$$

The characteristic frequencies of the reverse shock and the observed peak flux in Case II are

$$
\begin{aligned}
v_{\mathrm{a}, \mathrm{sd}}= & 1.9 \times 10^{11} \mathrm{~Hz}_{1}^{3 / 5} L_{0,49}^{11 / 5} M_{\mathrm{ej},-4}^{-2} \Gamma_{4,4} n^{19 / 50} \\
& \times f_{a}^{-1} f_{b}^{2 / 5}\left(\frac{\sigma}{1+\sigma}\right)^{1 / 5}, \\
v_{\mathrm{m}, \mathrm{sd}}= & 2.8 \times 10^{12} \mathrm{~Hz} L_{0,49}^{-7 / 2} T_{\mathrm{sd}, 3}^{-5} M_{\mathrm{ej},-4}^{4} \Gamma_{4,4}^{2} n^{1 / 2} \\
\times & \left(\frac{p-2}{p-1}\right)^{2} f_{a}^{2} f_{b} \sqrt{\frac{\sigma}{(1+\sigma)^{5}}}, \\
v_{\mathrm{c}, \mathrm{sd}}= & 5.5 \times 10^{15} \mathrm{~Hz} L_{0,49}^{13 / 2} T_{\mathrm{sd}, 3}^{9} M_{\mathrm{ej},-4}^{8} n^{-9 / 10} f_{b}^{-3}\left(\frac{1+\sigma}{\sigma}\right)^{3 / 2} \\
F_{v, \text { max }, \mathrm{sd}}= & 3.6 \times 10^{5} \mathrm{mJy} L_{0,49}^{-1 / 2} T_{\mathrm{sd}, 3}^{-2} M_{\mathrm{ej},-4}^{2} \Gamma_{4,4}^{-1} D_{27}^{-2} f_{b} \frac{\sqrt{\sigma}}{(1+\sigma)^{3 / 2}} .
\end{aligned}
$$


Case III: $M_{\mathrm{ej}, \mathrm{c}}<M_{\mathrm{ej}}$, or $T_{\mathrm{sd}}<T_{\mathrm{dec}}$. We take $L_{0}=10^{49} \mathrm{erg} \mathrm{s}^{-1}$ and $M_{\mathrm{ej}}=10^{-3} M_{\odot}$. Similar to Case I and Case II, we have (Gao et al. 2013; Wang \& Dai 2013; Wang et al. 2015)

$$
\begin{aligned}
& T_{\mathrm{N} 1}=2.07 \times 10^{-3} \text { days } L_{0,49}^{-1} M_{\mathrm{ej},-3}, \\
& T_{\mathrm{sd}}=2.3 \times 10^{-2} \text { days } L_{0,49}^{-1}, \\
& T_{\mathrm{dec}}=5.76 \text { days } L_{0,49}^{-7 / 3} T_{\mathrm{sd}, 3}^{-7 / 3} M_{\mathrm{ej},-3}^{8 / 3} n^{-1 / 3}\left(\frac{\sigma+2}{\sigma+1}\right)^{7 / 3}, \\
& T_{\mathrm{N} 2}=28.58 \text { days } L_{0,49}^{1 / 3} T_{\mathrm{sd}, 3}^{1 / 3} n^{-1 / 3}\left(\frac{\sigma+2}{\sigma+1}\right)^{-1 / 3}, \\
& \Gamma_{\mathrm{dec}}=5.59 L_{0,49} T_{\mathrm{sd}, 3} M_{\mathrm{ej},-4}^{-1}\left(\frac{\sigma+2}{\sigma+1}\right)^{-1}+1
\end{aligned}
$$

and the break frequencies and peak flux of Region 3 at $T_{\text {sd }}$ are derived as

$$
\begin{aligned}
v_{\mathrm{a}, \mathrm{sd}}= & 1.7 \times 10^{9} \mathrm{~Hz}_{1}^{3 / 5} L_{0,49}^{11 / 5} T_{\mathrm{sd}, 3}^{11 / 5} M_{\mathrm{ej},-3}^{-2} \Gamma_{4,4} n^{3 / 5} \\
& \times f_{a}^{-1} f_{b}^{2 / 5}\left(\frac{p-1}{p-2}\right)\left(\frac{\sigma}{1+\sigma}\right)^{1 / 5}, \\
v_{\mathrm{m}, \mathrm{sd}}= & 2.2 \times 10^{16} \mathrm{~Hz} L_{0,49}^{-7 / 2} T_{\mathrm{sd}, 3}^{-5} M_{\mathrm{ej},-3}^{4} \Gamma_{4,4}^{2} n^{1 / 2} \\
\times & f_{a}^{2} f_{b}\left(\frac{p-2}{p-1}\right)^{2} \sqrt{\frac{\sigma}{(1+\sigma)^{5}}}, \\
v_{\mathrm{c}, \mathrm{sd}}= & 2.7 \times 10^{10} \mathrm{~Hz} L_{0,49}^{-3 / 2} T_{\mathrm{sd}, 3}^{-3} M_{\mathrm{ej},-3}^{2} f_{b}\left(\frac{\sigma}{1+\sigma}\right)^{1 / 2}, \\
F_{v, \text { max }, \mathrm{sd}}= & 3.5 \times 10^{7} \mathrm{mJy} L_{0,49}^{-1 / 2} T_{\mathrm{sd}, 3}^{-2} M_{\mathrm{ej},-3}^{2} \Gamma_{4,4}^{-1} n^{1 / 5} D_{27}^{-2} \\
& \times f_{b} \frac{\sqrt{\sigma}}{(1+\sigma)^{3 / 2}} \cdot
\end{aligned}
$$

In Figs. 1 to 3, we can find that the dynamic evolutions of the shocks are only slightly dependent on the magnetar wind magnetization, but the characteristic frequencies and light curves of the reverse shock would be affected by the $\sigma$-value. The other parameters such as $M_{\mathrm{ej}}, n$, and $L_{0}$ could also play an important role in the brightness and evolution of the afterglow. In our calculation, we take the external medium density $n=1 \mathrm{~cm}^{-3}$.

\section{Discussions and conclusions}

Long-lasting energy injection from a post-merger millisecond magnetar wind has been proposed to drive multicomponent electromagnetic counterparts to gravitational wave bursts (Gao et al. 2013, 2015; Wang \& Dai 2013; Wang et al. 2015; Yu et al. 2013; Metzger \& Piro 2014). Current models concentrate on two extreme cases. In the first case, a pure Poynting flux drives the forward shock into the ambient medium (Gao et al. 2013; Wu et al. 2014). This is the $\sigma \rightarrow \infty$ extreme case. In the other extreme case, i.e., $\sigma \rightarrow 0$, the Poynting flux within the magnetosphere of the magnetar is transformed into kinetic energy of leptons by magnetic reconnection (Wang \& Dai 2013; Wang et al. 2015). We suggest that in most cases the magnetar wind is more likely to be a mixture of Poynting flux and ultra-relativistic $e^{ \pm}$pairs.

The comoving magnetic field strength in the emission regions is a critical parameter for the synchrotron radiation. For Region 2 (the forward-shocked medium), because the ambient medium is usually unmagnetized, the downstream magnetic field may be produced by the relativistic two-stream instability
(Medvedev \& Loeb 1999). This magnetic field strength is quantified by the equipartition parameter $\varepsilon_{B, f}$, i.e., the ratio of magnetic energy density to the total energy density behind the forward shock. The strength of this magnetic field is quite low for $\varepsilon_{B, f}$ in the range $10^{-4}-10^{-2}$, as inferred by fitting multiwavelength afterglows of GRBs (Panaitescu \& Kumar 2002; Yost et al. 2003). In addition, this magnetic field may be smallscale and random.

The magnetic field in the reverse-shocked region could be quite different because its origin depends on the magnetization of the upstream cold wind. Wang \& Dai (2013) and Wang et al. (2015) assumed that the upstream fluid of the reverse shock is unmagnetized. To calculate synchrotron radiation from Region 3 , a random magnetic field is introduced through the equipartition parameter $\varepsilon_{B, r}$, which can be written as

$$
B_{3, \mathrm{ra}}^{\prime}=\sqrt{8 \pi \varepsilon_{B, r} e_{3}}=\sqrt{8 \pi \varepsilon_{B, r}\left(4 \Gamma_{34}+3\right)\left(\Gamma_{34}-1\right) n_{4}^{\prime} m_{e} c^{2}} .
$$

We suggest that the magnetization of the unshocked wind could be so large that the magnetic field in the reverse-shocked region is amplified by compressing the upstream magnetic field. The strength of this magnetic field is dependent on the magnetization parameter $\sigma$. Because the field of shock-compressed upstream magnetic field is globally structured, the reverse shock emission may have a high polarization.

In all dynamical cases, when $t>T_{\mathrm{dec}}$ the dynamic evolution of the blast wave obeys the self-similar solution of Blandford \& McKee (1976). The evolution of the blast wave depends on the total energy $E$ and the external medium density $n$ and is almost independent of the magnetar wind magnetization when $t>T_{\text {dec }}$ (see Figs. 1-3). A similar result has been found in previous works (Emmering \& Chevalier 1987; Bucciantini 2002; Mimica et al. 2009, 2010; Mao et al. 2010).

The reverse shock emission is influenced by the degree of magnetization. On the one hand, a weak magnetic field (in the low- $\sigma$ regime case) would suppress the synchrotron radiation. The formation of a reverse shock always takes place in a weakly magnetized wind as long as the shocked wind could move supersonically with respect to the unshocked wind. On the other hand, a strong magnetic field (in the high- $\sigma$ regime case) would reduce the energy fraction of the shock-accelerated electrons. As shown in Fig. 4, the peak flux of reverse shock emission is a function of $\sigma$. We find that $\sigma \sim 0.3$ leads to the strongest reverse shock emission.

It is important to note the difference between the magnetized wind and the magnetized ejecta. For the latter case where the composition is baryons, Mimica et al. (2010) show that the RS emission peaks for $\sigma \sim 0.1$ (their Figs. 3 and 6). It is also shown that for $\sigma \sim 1$ the RS emission should disappear, while Giannios et al. (2008) shows that even for $\sigma \sim 0.3$ the RS may vanish for some combinations of the wind luminosity and the external medium density. The disappearance of RS in this case is linked to the low relative Lorentz factor between Region 3 and Region 4, as can be seen in the lower panel of Fig. 3 in Mimica et al. (2009). For the magnetized magnetar wind that is the focus of this paper, for typical parameters of the ejecta and the magnetar, e.g., $\Gamma_{w}>10^{4}$, the maximum Lorentz factor of the blast wave is $\Gamma \sim 10$, which means that $\Gamma_{34} \approx \Gamma_{w} / 2 \Gamma \gg 1$ is always satisfied. This is also true for the prerequisite $\Gamma_{34} \gg f_{a} f_{b} f_{c}$ as determined by Zhang \& Kobayashi (2005) because $f_{a}<1$, $f_{b}<1$, and $f_{c} \sim 10$. A strong reverse shock would therefore exist, even in the high magnetization regime.

Figures 1-3 show that the light curves for different values of the magnetization parameter are quite similar. Consequently, 
L. D. Liu et al.: Reverse shock emission driven by post-merger millisecond magnetar winds

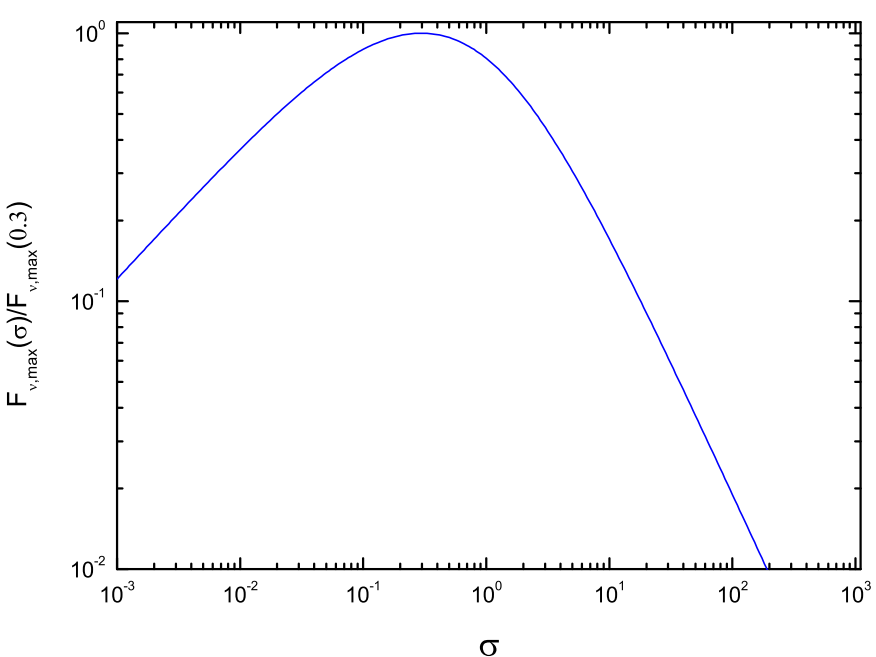

Fig. 4. Peak flux of the reverse shock emission as a function of $\sigma$. It can be seen that the $\sigma \sim 0.3$ leads to the strongest reverse shock emission. The emission of magnetized lepton-dominated wind as a function of magnetization is quite different from that of magnetized baryondominated ejecta. See the main text for more details.

it is not easy to measure magnetization directly from light curves. To measure the magnetization, polarization should be measured. The successful polarization measurement of the optical afterglow emission of GRB090102 obtains a linear polarization degree of about $10 \%$ (Steele et al. 2009). It indicates largescale magnetic fields in the emission region (Steele et al. 2009; Mimica et al. 2010; Harrison \& Kobayashi 2013).

Acknowledgements. This work is supported by the National Basic Research Program ("973" Program) of China under Grant No. 2014CB845800 and the National Natural Science Foundation of China (grant Nos. 11573014 and 11473008).

\section{References}

Abadie, J., Abbott, B. P., Abbott, R., et al. 2010, CQG, 27, 173001 Atoyan, A. M. 1999, A\&A, 346, L49

Barthelmy, S. D., Chincarini, G., Burrows, D. N., et al. 2005, Nature, 438, 994

Bartos, I., Brady, P., \& Márka, S. 2013, CQGra, 30, 123001

Berger, E., Fong, W., \& Chornock, R. 2013, ApJ, 774, L23

Blandford, R. D., \& McKee, C. F. 1976, Physics of Fluids, 19, 1130

Bucciantini, N. 2002, A\&A, 387, 1066

Coroniti, F. V. 1990, ApJ, 349, 538
Dai, Z. G. 2004, ApJ, 606, 1000

Dai, Z. G., \& Lu, T. 1998a, A\&A, 333, L87

Dai, Z. G., \& Lu, T. 1998b, Phys. Rev. Lett., 81, 4301

Dai, Z. G., Wang, X. Y., Wu, X. F., \& Zhang, B. 2006, Science, 311, 1127

Duncan, R. C., \& Thompson, C. 1992, ApJ, 392, L9

Eichler, D., Livio, M., Piran, T., \& Schramm, D. N. 1989, Nature, 340, 126

Emmering, R. T., \& Chevalier, R. A. 1987, ApJ, 321, 334

Fan, Y. Z., Wei, D. M., \& Wang, C. F. 2004, A\&A, 424, 477

Fox, D. B., Frail, D. A., Price, P. A., et al. 2005, Nature, 437, 845

Gao, H., Ding, X., Wu, X. F., Zhang, B., \& Dai, Z. G. 2013, ApJ, 771, 86

Gao, H., Ding, X., Wu, X.-F., Dai, Z.-G., \& Zhang, B. 2015, ApJ, 807, 163

Gehrels, N., Sarazin, C. L., O’Brien, P. T., et al. 2005, Nature, 437, 851

Giacomazzo, B., \& Perna, R. 2013, ApJ, 771, L26

Giannios, D., Mimica, P., \& Aloy, M. A. 2008, A\&A, 478, 747

Harrison, R., \& Kobayashi, S. 2013, ApJ, 772, 101

Hotokezaka, K., Kiuchi, K., Kyutoku, K., et al. 2013, Phys. Rev. D, 87, 024001

Huang, Y. F., Dai, Z. G., \& Lu, T. 1999, MNRAS, 309, 513

Kennel, C. F., \& Coroniti, F. V., 1984, ApJ, 283, 694

Kulkarni, S. R. 2005 [arXiv: astro-ph/0510256]

Li, L.-X., \& Paczyński, B. 1998, ApJ, 507, L59

Lyutikov, M. 2011, MNRAS, 411, 422

Mao, Z., Yu, Y. W., Dai, Z. G., Pi, C. M., \& Zheng, X. P. 2010, A\&A, 518, A27

Medvedev, M. V., \& Loeb, A. 1999, ApJ, 526, 697

Metzger, B. D., \& Berger, E. 2012, ApJ, 746, 48

Metzger, B. D., \& Piro, A. L. 2014, MNRAS, 439, 3916

Metzger, B. D., Giannios, D., Thompson, T. A., Bucciantini, N., \& Quataert, E. 2011, MNRAS, 413, 2031

Michel, F. C. 1994, ApJ, 431, 397

Michel, F. C., \& Li, H. 1999, Phys. Rep., 318, 227

Mimica, P., Giannios, D., \& Aloy, M. A. 2009, A\&A, 494, 879

Mimica, P., Giannios, D., \& Aloy, M. A. 2010, MNRAS, 407, 2501

Mizuno, Y., Zhang, B., Giacomazzo, B., et al. 2009, ApJ, 690, L47

Nakar, E., \& Piran, T. 2011, Nature, 478, 82

Paczyński, B. 1986, ApJ, 308, L43

Panaitescu, A, \& Kumar, P. 2002, ApJ, 571, 779

Piran, T., Nakar, E., \& Rosswog, S. 2013, MNRAS, 430, 2121

Rezzolla, L., Baiotti, L., Giacomazzo, B., et al. 2010, CQG, 27, 114105

Rosswog, S. 2005, ApJ, 634, 1202

Rosswog, S., Piran, T., \& Nakar, E. 2013, MNRAS, 430, 2585

Rowlinson, A., O’Brien, P. T., Tanvir, N. R., et al. 2010, MNRAS, 409, 531

Rowlinson, A., O'Brien, P. T., Metzger, B. D., et al. 2013, MNRAS, 430, 1061

Sari, R., Piran, T., \& Narayan, R. 1998, ApJ, 497, L17

Steele, I. A., Mundell, C. G., Smith, R. J., Kobayashi, S., \& Guidorzi, C. 2009, Nature, 462, 767

Tanvir, N. R., Levan, A. J., Fruchter, A. S., et al. 2013, Nature, 500, 547

Wang, L. J., \& Dai, Z. G. 2013, ApJ, 774, L33

Wang, L. J., Dai, Z. G. Yu, Y. W. 2015, ApJ, 800, 79

Wu, X. F., Dai, Z. G., Huang, Y. F., \& Lu, T. 2003, MNRAS, 342, 1131

Wu, X.-F., Gao, H., Ding, X., et al. 2014, ApJ, 781, L10

Yost, S., Harrison, F. A., Sari, R., \& Frail, D. A. 2003, ApJ, 597, 459

Yu, Y. W., \& Dai, Z. G. 2007, A\&A, 470, 119

Yu, Y. W., Zhang, B., \& Gao, H. 2013, ApJ, 776, L40

Zhang, B. 2013, ApJ, 763, L22

Zhang, B., \& Kobayashi, S. 2005, ApJ, 628, 315

Zhang, B., \& Mészáros, P., 2001, ApJ, 552, L35 